\title{
Simple K3 Singularities Which Are Hypersurface Sections of Toric Singularities
}

By

\author{
Hiroyasu Tsuchihashi*
}

\section{Introduction}

Yonemura [9] classified the weights of non-degenerate quasi-homogeneous polynomials on $C^{4}$ which define simple $\mathrm{K} 3$ singularities. On the other hand, to each quasi-homogeneous polynomial $f=\Sigma_{\nu \in(Z \geqq 0)}{ }^{4} c_{v} z^{v}$ there exists an element $u_{0}$ in $\left(\boldsymbol{Q}_{>0}\right)^{4}$ such that $\left\langle v, u_{0}\right\rangle=1$ if $c_{v} \neq 0$, where $z^{\left(m_{1}, m_{2}, m_{3}, m_{+}\right)}=z_{1}^{m_{1}} z_{2}^{m_{2}} z_{3}^{m_{3}} z_{4}^{m_{4}}$. Then we may regard the point $u_{0}$ as the weight of $f$. Let $\Delta^{*}$ be the convex hull of $\left\{v \in\left(Z_{\geqq 0}\right)^{4} \mid\left\langle v, u_{0}\right\rangle=1\right\}$. Then $\operatorname{dim} \Delta^{*}=3$ and $(1,1,1,1) \in \operatorname{Int}\left(\Delta^{*}\right)$, if $f$ defines a simple K3 singularity (see [9]). As a generalization of this fact, we obtain:

Theorem. Let $f$ be a non-degenerate holomorphic function on the toric singularity $Y=\operatorname{Spec} C\left[\sigma^{*} \cap\left(Z^{4}\right)^{*}\right]$ with $f(y)=0$ and let $X=\{f=0\}$, where $\sigma^{*}$ is the dual cone of a 4-dimensional strongly convex cone $\sigma$ in $\boldsymbol{R}^{4}$ generated by primitive elements $u_{1}, u_{2}, \ldots$ and $u_{s}$ in $Z^{4}$ and $\{y\}=\left\{x \in Y \mid z^{v}(x)=0\right.$ for any $\left.v \in\left(\sigma^{*} \cap\left(Z^{4}\right)^{*}\right) \backslash\{0\}\right\}$. If $(X, y)$ is a simple $K 3$ singularily, then the following two conditions are satisfied.

(1) $Y$ is Gorenstein, i.e., there exists an element $v_{0} \in\left(Z^{4}\right)^{*}$ such that $\left\langle v_{0}, u_{i}\right\rangle=1$, if $R_{\geqq 0} u_{i}$ is a 1-dimensional face of $\sigma$, for $i=1$ through $s$.

(2) There exists an element $u_{0} \in \operatorname{Int}(\sigma)$ such that $f=\Sigma_{v \in \sigma^{1}} \cap\left(Z^{4}\right)$, $c_{1} z^{v}$ with $c_{v}=0$ if $\left\langle v, u_{0}\right\rangle<1$, that $\operatorname{dim} \Delta^{*}=3$ and that $v_{0} \in \operatorname{Int}\left(\Delta^{*}\right)$, where $\Delta^{*}$ is the convex hull of $\left\{v \in \sigma^{*} \cap\left(\mathbb{Z}^{4}\right)^{*} \mid\left\langle v, u_{0}\right\rangle=1\right\}$.

The purpose of this paper is to show that the pairs $\left(\sigma, u_{0}\right)$ satisfying the conditions of the above theorem are finite modulo $\mathrm{GL}(4, \mathbb{Z})$. Moreover, all representatives of them are obtained by an algorithm which can be excuted by a computer. (However, the program I wrote spent so much time that I could not wait to the end. The number of the equivalent classes is at least greater than 10000.)

Communicated by K. Saito, June 25, 1990.

* 1991 Mathematics Subject Classification: 32C40.

Faculty of Liberal Arts, Tohoku Gakuin University, Sendai 981-31, Japan 
In $\S 1$, we prove the above theorem and show that there exists a partial order on the set of the pairs satisfying the conditions of the above theorem such that for a pair $\left(\sigma, u_{0}\right)$, all the pairs $\left(\tau, u_{0}\right) \geqq\left(\sigma, u_{0}\right)$ are finite and obtained by a simple algorithm (see Proposition 1.6 and its proof).

In $\S 2$, we classify "minimal" pairs into some classes.

In $\S 3$, we enumerate all pairs belonging to each of the classes and make a list of representatives of them at the end of this paper.

\section{§1. Toric Singularities and Their Hypersurface Sections}

Let $N=\mathbb{Z}^{n+1}$ be a free $\mathbb{Z}$-module of rank $n+1 \geqq 3$ and let $N^{*}$ be its dual module with canonical pairing $\langle\rangle:, N^{*} \times N \rightarrow \mathbb{Z}$. Let $\sigma=\mathbb{R}_{\geqq 0} u_{1}+\mathbb{R}_{\geqq 0} u_{2}+\ldots+$ $\mathbb{R}_{\geqq 0} u_{s}$ be an $(n+1)$-dimensional strongly convex rational cone in $N_{\mathbb{R}}:=N \otimes_{Z} \mathbb{R}$ generated by primitive elements $u_{i}$ in $N$. Here we may assume that $\mathbb{R}_{\geqq 0} u_{i}$ is a 1-dimensional face of $\sigma$, i.e., there exists an element $v$ in $N_{\mathbb{R}}^{*}$ such that $\{u \in$ $\sigma \mid\langle v, u\rangle=0\}=\mathbb{R}_{\geqq 0} u_{i}$ for each $i=1$ through $s$. Let $Y=\operatorname{Spec} C\left[\sigma^{*} \cap N^{*}\right]$ and let $z^{v}: Y \rightarrow \mathbb{C}$ be the character of $v$, which is the natural extension of $v \otimes 1 c^{\times}$: Spec $\mathbb{C}\left[N^{*}\right] \simeq\left(\mathbb{C}^{\times}\right)^{n+1} \rightarrow \mathbb{C}^{\times}$, for each $\mathrm{v}$ in $\sigma^{*} \cap N^{*}$. Then the set $\left\{x \in Y \mid z^{v}(x)=\right.$ 0 for all $\left.v \in\left(\sigma^{*} \cap N^{*}\right) \backslash\{0\}\right\}$ consists of only one point $y$ and any holomorphic function $f$ on $Y$ with $f(y)=0$ is expressed as the power series:

$$
f:=\Sigma_{\nu \in\left(\sigma^{+} \cap N^{*}\right) \backslash\{0\}} c_{v} z^{v} .
$$

Let $X$ be a hypersurface section of $Y$ containing $y$, i.e., $X=\{f=0\}$, for a holomorphic function $f$ on $Y$ with $f(y)=0$. Here we note that if $(X, y)$ is an isolated singularity, then the dimension of the singular locus $\operatorname{Sing}(Y)$ of $Y$ is not greater than 1 , i.e., any $(n-1)$-dimensional face of $\sigma$ is generated by a part of a basis of $N$. Assume that $X$ is normal and that $X \backslash\{y\}$ has only rational singularities. Then by [7] and [1], we obtain:

Proposition 1.1. The following three conditions are equivalent.

(1) $(X, y)$ is Gorenstein.

(2) $(Y, y)$ is Gorenstein.

(3) $(G)$ There exists an element $v_{0} \in N^{*}$ such that $\left\langle v_{0}, u_{i}\right\rangle=1$ for $1 \leqq i \leqq s$.

We denote the above $v_{0}$, by $v(\sigma)$.

Definition 1.2. The Newton polyhedron $\Gamma_{+}(f)$ of $f$ is the convex hull of $\cup_{c_{v} \neq 0}\left(v+\sigma^{*}\right)$ and the Newton boundary $\Gamma(f)$ of $f$ is the union of the compact faces of $\Gamma_{+}(f)$.

Definition 1.3. We call $f$ non-degenerate, if $\partial f_{\Delta^{*}} / \partial z_{1}=\ldots=\partial f_{\Delta^{*}} / \partial z_{\mathbf{n}+1}=$ 0 has no solutions in $T:=\operatorname{Spec} C\left[N^{*}\right] \subset Y$ for each face $\Delta^{*}$ of $\Gamma_{+}(f)$, where $f_{\Delta^{*}}=\Sigma_{\nu \in \Delta^{*} \cap N^{*}} c_{v} z^{\nu}$ and $\left(z_{1}, z_{2}, \ldots, z_{\mathrm{n}+1}\right)$ is a global coordinate of $T$, i.e., $z_{i}=$ 
$z^{v_{t}}$ for a basis $\left\{v_{1}, v_{2}, \ldots, v_{\mathrm{n}+1}\right\}$ of $N^{*}$.

Proposition 1.4. ([6, Theorem 2.2]) Assume that the condition $(G)$ in Proposition 1.1 is satisfied and that $f$ is non-degenerate. Then $(X, y)$ is purely elliptic if and only if $v(\sigma) \in \Gamma(f)$. (See [8], for the definition of a purely elliptic singularity.)

Remark. If $v(\sigma) \in \partial \Gamma_{+}(f) \backslash \Gamma(f)$, then $X \backslash\{y\}$ has irrational singularities.

Proposition 1.5. Under the assumption of Proposition 1.4, $(X, y)$ is of $(0, n-1)$-type if and only if $\operatorname{dim} \Delta^{*}=n$, where $\Delta^{*}$ is the face of $\Gamma(f)$ with $v(\sigma) \in$ $\operatorname{Int}\left(\Delta^{*}\right)$. (See [2], for the definition of $(0, i)$-type of a purely elliptic singularity.)

Proof. Let $\Sigma$ be a subdivision of the dual Newton decomposition $\Gamma^{*}(f)$ of $\Gamma(f)$ consisting of non-singular cones (see [5] and [6], for the definition of $\left.\Gamma^{*}(f)\right)$. Then $\tilde{Y}:=T_{N} \operatorname{emb}(\Sigma)$ and $\tilde{X}$ are non-singular, where $\tilde{X}$ is the proper transformation of $X$ under the holomorphic map $P: \tilde{Y} \rightarrow Y$ obtained by the morphism of r.p.p. decompositions $(N, \Sigma) \rightarrow(N$, f faces of $\sigma\})$. Let $\Sigma_{1}$ be the set of the 1-dimensional cones in $\Sigma$ which are not 1-dimensional faces of $\sigma$ and let $E_{\tau}$ be the intersection of the closure of orb $(\tau)$ with $\tilde{X}$, for each $\tau$ in $\Sigma_{1}$. Then $P_{\mid \tilde{X}^{-1}}(y)=\Sigma_{\tau \in \Sigma_{0}} E_{\tau}$, where $\Sigma_{0}=\left\{\tau \in \Sigma_{1} \mid \tau \subset \operatorname{Int}(\sigma) \cup\{0\}\right\}$ and we can express $K_{\tilde{X}}=\left(P_{\mid \tilde{X}}\right)^{*} K_{X}+\Sigma_{\tau \in \Sigma_{1}} \mathrm{a}_{\tau} E_{\tau}$. Here we note that $a_{\tau}=\left\langle v(\sigma), u_{\tau}\right\rangle-d\left(u_{\tau}\right)-1$, by $[6$, Lemma 2.1], where $u_{\tau}$ is the primitive element in $N$ generating $\tau$ and $d\left(u_{\tau}\right)=\min$ $\left\{\left\langle v, u_{\tau}\right\rangle \mid v \in \Gamma_{+}(f)\right\}$. Hence $a_{\tau} \geqq-1$, for each $\tau$ in $\Sigma_{1}$. Assume that $\operatorname{dim} \Delta^{*}=n$. Then there exists only one 1-dimensional cone $\tau$ in $\Sigma_{0}$ with $a_{\tau}=-1$ and $E_{\tau}$ is irreducible. Hence $(X, y)$ is of $(0, n-1)$-type. Next, assume that $\operatorname{dim} \Delta^{*} \leqq n-1$. Then we easily see that there exist at least two 1-dimensional cones $\tau$ in $\Sigma_{1}$ such that $a_{\tau}=-1$ and that $E_{\tau} \neq \varnothing$. Hence $(X, y)$ is not of $(0, n-1)$-type. q.e.d.

Assume that $f$ is a non-degenerate holomorphic function on $Y$ with $f(y)=0$ and let $X=\{f=0\}$. When $n=3,(X, y)$ is a simple $\mathrm{K} 3$ singularity (i.e., $(X, y)$ is Gorenstein purely elliptic of $(0,2)$-type [3]), if and only if $(Y, y)$ is Gorenstein and $v(\sigma)$ is contained in the interior of a 3-dimensional face of $\Gamma(f)$, by Propositions $1.1,1.4$ and 1.5. Assume that $(X, y)$ is Gorenstein purely elliptic of $(0, n-1)$-type. Then there exists the unique element $u_{0}$ in Int $(\sigma)$ such that $\left\langle v, u_{0}\right\rangle=1$ for all elements $v$ in the face $\Delta^{*}$ of $\Gamma(f)$ whose interior contains $v(\sigma)$. Hence $\Delta^{*}$ is contained in

$$
\Delta_{\sigma}^{*}\left(u_{0}\right):=\text { convex hull of }\left\{v \in \sigma^{*} \cap N^{*} \mid\left\langle v, u_{0}\right\rangle=1\right\} .
$$

Therefore, the pair $\left(\sigma, u_{0}\right)$ satisfies the following condition:

$$
\text { (E) } \operatorname{dim} \Delta_{\sigma}^{*}\left(u_{0}\right)=n \text { and } v(\sigma) \in \operatorname{Int}\left(\Delta_{\sigma}^{*}\left(u_{0}\right)\right) \text {. }
$$

Thus we obtain the theorem in Introduction. Conversely, assume that $\left(\sigma, u_{0}\right)$ 
satisfies the conditions $(\mathrm{G})$ and $(\mathrm{E})$, and let $X=\{f=0\}$, where $f=$ $\Sigma_{v \in \Delta_{\sigma}^{*}\left(u_{0}\right) \cap N^{*}} c_{v} z^{v}+$ higher terms, for certain non-zero complex numbers $c_{\nu}$. Then $(X, y)$ is Gorenstein purely elliptic of $(0, n-1)$-type, if $f$ is non-degenerate and $(X, y)$ is an isolated singularity. Let

$$
\begin{gathered}
\widetilde{\mathscr{E}} \mathrm{n}=\left\{\left(\sigma, u_{0}\right) \mid \sigma \text { is an }(n+1)\right. \text {-dimensional strongly convex rational cone } \\
\text { satisfying } \left.(\mathrm{G}), u_{0} \in \operatorname{Int}(\sigma) \text { and } u_{0} \text { satisfies }(\mathrm{E})\right\}
\end{gathered}
$$

and let $\mathscr{E}^{n}=\tilde{\mathscr{E}}^{n} / \sim$, where $\left(\sigma, u_{0}\right) \sim\left(\sigma^{\prime}, u_{0}^{\prime}\right)$ if and only if there exists an element $g$ in GL(N) such that $g \sigma=\sigma^{\prime}$ and that $g\left(u_{0}\right)=u_{0}^{\prime}$. We define a partial order on $\mathscr{\mathscr { g }}^{n}$ as follows: $\left(\sigma, u_{0}\right) \geqq\left(\sigma^{\prime}, u_{0}^{\prime}\right)$ if and only if $\sigma \supset \sigma^{\prime}, v(\sigma)=v\left(\sigma^{\prime}\right)$ and $u_{0}=u_{0}^{\prime}$. Let

$$
\widetilde{\mathscr{E}}_{0}^{n}=\left\{\left(\sigma, u_{0}\right) \in \tilde{\mathscr{E}}^{n} \mid\left(\sigma, u_{0}\right) \text { is minimal }\right\}
$$

and let $\mathscr{E}_{0}^{n}=\widetilde{\mathscr{E}}_{0}^{n} / \sim$, where we call $\left(\sigma, u_{0}\right)$ minimal, if $\left(\sigma, u_{0}\right) \geqq\left(\tau, u_{0}\right)$ implies $\left(\sigma, u_{0}\right)=\left(\tau, u_{0}\right)$, for any $\left(\tau, u_{0}\right) \in \widetilde{\mathscr{C}}^{n}$.

Remark. (1) Assume that $\left(\sigma, u_{0}\right) \in \widetilde{\mathscr{E}}^{n}$. If the cone $\tau$ generated by a subset of $L:=\{u \in \sigma \cap N \mid\langle v(\sigma), u\rangle=1\}$ is $(n+1)$-dimensional strongly convex and contains $u_{0}$ in the interior, then $\left(\tau, u_{0}\right) \in \widetilde{\mathscr{C}}^{n}$, because $\tau^{*} \supset \sigma^{*}$.

(2) Since $\# L<+\infty$, for any pair $\left(\sigma, u_{0}\right)$ in $\widetilde{\mathscr{g}}^{n}$, we have $\#\left\{\left(\tau, u_{0}\right) \in \widetilde{\mathscr{E}}^{n} \mid\left(\sigma, u_{0}\right)\right.$ $\left.\geqq\left(\tau, u_{0}\right)\right\}<+\infty$. Hence for any pair $\left(\sigma, u_{0}\right)$ in $\widetilde{\mathscr{E}}^{n}$, there exists a pair $\left(\tau, u_{0}\right)$ in $\widetilde{\mathscr{E}}_{0}^{n}$ with $\left(\sigma, u_{0}\right) \geqq\left(\tau, u_{0}\right)$.

Let $C\left(\sigma, u_{0}\right)=\left\{\left(\tau, u_{0}\right) \in \widetilde{\mathscr{g}}^{n} \mid\left(\tau, u_{0}\right) \geqq\left(\sigma, u_{0}\right)\right\}$, for a pair $\left(\sigma, u_{0}\right)$ in $\widetilde{\mathscr{E}}^{n}$. Then by the above remark, we have $\widetilde{\mathscr{E}}^{n}=\cup_{\left(\sigma, u_{0}\right) \in \mathscr{\mathscr { C }}_{0}^{n}} C\left(\sigma, u_{0}\right)$. Hence if $\mathscr{E}_{0}^{n}$ is a finite set, then so is $\mathscr{E}^{n}$, by the following proposition.

Proposition 1.6. $C\left(\sigma, u_{0}\right)$ is a finite set, for any pair $\left(\sigma, u_{0}\right)$ in $\widetilde{\mathscr{E}}^{n}$.

Proof. Since for any pair $\left(\tau, u_{0}\right)$ in $C\left(\sigma, u_{0}\right), \Delta_{\tau}^{\dagger}\left(u_{0}\right)$ is the convex hull of a

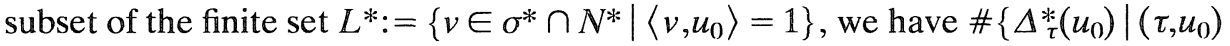
$\left.\in C\left(\sigma, u_{0}\right)\right\}<+\infty$. Conversely, let $\Delta^{*}$ be the convex hull of a subset of $L^{*}$ such that $v(\sigma) \in \operatorname{Int}\left(\Delta^{*}\right)$ and that $\operatorname{dim} \Delta^{*}=n$. Then $\#\left\{u \in\left(\mathbb{R}_{\geqq 0} \Delta^{*}\right)^{*} \cap N \mid\langle v(\sigma), u\rangle\right.$ $=1\}<+\infty$. Hence $C^{\prime}:=\left\{\left(\tau, u_{0}\right) \in C\left(\sigma, u_{0}\right) \mid \Delta_{\tau}^{\prime}\left(u_{0}\right)=\Delta^{*}\right\}$ is a finite set, because $\tau \subset\left(\mathbb{R}_{\geqq 0} \Delta^{*}\right)^{*}$ for any pair $\left(\tau, u_{0}\right)$ in $C^{\prime}$. Therefore, $C\left(\sigma, u_{0}\right)$ is a finite set.

q.e.d.

Next, we show that for a cone $\sigma$ satisfying the condition $(G)$, all the elements $u_{0}$ in $\operatorname{Int}(\sigma)$ satisfying the condition $(\mathbb{E})$ are finite. Let $W_{\sigma}\left(v_{0}\right)=\{u \in$ $\left.\operatorname{Int}(\sigma) \mid \operatorname{dim} \Delta_{\sigma}^{+}(u)=n, \quad v_{0} \in \operatorname{Int}\left(\Delta_{\sigma}^{+}(u)\right)\right\}$, for an $(n+1)$-dimensional strongly convex rational cone $\sigma$ and for an element $v_{0}$ in $N_{R}^{+}$.

Theorem 1.7. $W_{\sigma}\left(v_{0}\right)$ is a finite set, for any $v_{0} \in \operatorname{Int}\left(\sigma^{*}\right)$. 
Proof. For $v_{1}, v_{2}, \ldots, v_{j} \in \sigma^{*} \cap N^{*}$, let $W\left(v_{1}, v_{2}, \ldots, v_{\mathrm{j}}\right)=\left\{u \in W_{\sigma}\left(v_{0}\right) \mid\right.$ $\left.\left\langle v_{1}, u\right\rangle=\cdots=\left\langle v_{j}, u\right\rangle=1\right\}$. For $u \in N_{R}$, let $W^{*}(u)=\left\{v \in \sigma^{*} \cap N^{*} \mid\langle v, u\rangle<1\right\}$. Here we note that if $u \in \operatorname{Int}(\sigma)$, then $W^{*}(u)$ is a finite set. First, take an element $u_{0} \in \operatorname{Int}(\sigma)$ with $\left\langle v_{0}, u_{0}\right\rangle=1$. Then for any element $u$ in $W_{\sigma}\left(v_{0}\right)$ with $u \neq u_{0}$, we see that $\left\{v \in W^{*}\left(u_{0}\right) \mid\langle v, u\rangle=1\right\} \neq \varnothing$. Hence $w_{\sigma}\left(v_{0}\right) \subset\left\{u_{0}\right\} \cup \cup_{v_{1} \in W^{+}\left(u_{0}\right)} W\left(v_{1}\right)$. Here we note that if $W\left(v_{1}\right) \neq \varnothing$, then $v_{0}$ and $v_{1}$ are linearly independent. Next, if $W\left(v_{1}\right) \neq \varnothing$, then we can take an element $u_{1} \in \operatorname{Int}(\sigma)$ with $\left\langle v_{0}, u_{1}\right\rangle=\left\langle v_{1}, u_{1}\right\rangle=1$, for each $v_{1} \in W^{*}\left(u_{0}\right)$. Then we have $W\left(v_{1}\right) \subset\left\{u_{1}\right\} \cup \cup_{v_{2} \in W^{*}\left(u_{1}\right)} W\left(v_{1}, v_{2}\right)$. Proceeding similarly, we finally obtain $W\left(v_{1}, \ldots, v_{n-1}\right) \subset\left\{u_{n-1}\right\} \cup \cup_{v_{n} \in W^{k}\left(u_{n-1}\right)}$ $W\left(v_{1}, \ldots, v_{n}\right)$. Then $\# W\left(v_{1}, \ldots, v_{n}\right) \leqq 1$, because $v_{0}, v_{1}, \ldots$ and $v_{\mathrm{n}}$ are linearly independent, if $W\left(v_{1}, \ldots, v_{n}\right) \neq \varnothing$. Hence $\# W\left(v_{1}, \ldots, v_{n-1}\right)<+\infty$ and thus $\# W_{\sigma}\left(v_{0}\right)<+\infty$.

q.e.d.

In the next section, the following proposition plays key role.

Proposition 1.8. If $W_{\sigma}\left(v_{0}\right) \neq \varnothing$ for an element $v_{0} \in \operatorname{Int}\left(\sigma^{*}\right)$, then \#IL $L_{\sigma}\left(v_{0}\right) \leqq$ 1 , where $I L_{\sigma}\left(v_{0}\right)=\left\{u \in \operatorname{Int}(\sigma) \cap N \mid\left\langle v_{0}, u\right\rangle=1\right\}$. Conversely, if $I L_{\sigma}\left(v_{0}\right)=\left\{u_{0}\right\}$, then $W_{\sigma}\left(v_{0}\right) \subset\left\{u_{0}\right\}$.

Proof. If $I L_{\sigma}\left(v_{0}\right) \neq \varnothing$, then for each element $u_{0}$ in $I L_{\sigma}\left(v_{0}\right)$, we have $\left\langle v, u_{0}\right\rangle$ $>0$ for any $v$ in $\sigma^{*} \backslash\{0\}$ and hence $\left\langle v, u_{0}\right\rangle \geqq 1$ for any $v$ in $\left(\sigma^{*} \backslash\{0\}\right) \cap N^{*}$. Therefore, $W_{\sigma}\left(v_{0}\right) \subset\left\{u_{0}\right\}$, as we see in the proof of Theorem 1.7. Hence if $\# I L_{\sigma}\left(\mathrm{v}_{0}\right) \geqq 2$, then $W_{\sigma}\left(v_{0}\right)=\emptyset$.

q.e.d.

\section{\$2. Classification}

We restrict ourselves to the case that $n=3$ and show that $\mathscr{E}_{0}^{3}$ is a finite set, in the rest of this paper. For finite elements $u_{1}, u_{2}, \ldots, u_{s}$ in $N_{R}$, we denote by $u_{1} u_{2} \ldots u_{s}$, the convex hull $\left\{a_{1} u_{1}+a_{2} u_{2}+\ldots+a_{s} u_{s} \mid a_{i} \geqq 0, a_{1}+a_{2}+\ldots+a_{\mathrm{s}}=\right.$ $1\}$ of $\left\{u_{1}, u_{2}, \ldots, u_{s}\right\}$.

Theorem 2.1. Any pair $\left(\sigma, u_{0}\right)$ in $\tilde{\mathscr{E}}_{0}^{3}$ is one of the following.

(1) $\sigma$ is generated by four primitive elements $u_{1}, u_{2}, u_{3}$ and $u_{4}$ in $N$ and $u_{l} u_{J} \cap N=$ $\left\{u_{i}, u_{j}\right\}$ for each $\{i, j\} \subset\{1,2,3,4\}$.

(2) $\sigma$ is generated by five primitive elements $u_{1}, u_{2}, \ldots$ and $u_{5}$ in $N, u_{0} \in \operatorname{Int}\left(u_{1} u_{2} u_{3}\right)$ and $u_{1} u_{2} u_{3} \cap u_{4} u_{5} \neq \varnothing$.

(3) $\sigma$ is generated by six primitive elements $u_{1}, u_{2}, \ldots$ and $u_{6}$ in $N, u_{0} \in \operatorname{Int}\left(u_{1} u_{2}\right)$, Int $\left(u_{3} u_{4}\right)$, Int $\left(u_{5} u_{6}\right)$ and $u_{1} u_{2} \ldots u_{6} \cap N \subset\left\{u_{0}, u_{1}, \ldots, u_{6}\right\}$.

Proposition 2.2. $\left(\sigma, u_{0}\right)$ in (1) of Theorem 2.1 is one of the following.

$(1-1) \quad u_{1} u_{2} u_{3} u_{4} \cap N \subset\left\{u_{0}, u_{1}, u_{2}, u_{3}, u_{4}\right\}$.

$(1-2) \quad\left(u_{1} u_{2} u_{3} u_{4} \cap N\right) \backslash\left\{u_{0}, u_{1}, u_{2}, u_{3}, u_{4}\right\}=\left\{u_{5}, \ldots, u_{s}\right\} \subset u_{i} u_{j} u_{k}(s \geqq 5)$ and $u_{0} \in$ $u_{s} u_{k} u_{l}$, where $u_{5}=\frac{1}{3}\left(u_{l}+u_{j}+u_{6}\right), u_{6}=\frac{1}{2}\left(u_{5}+u_{7}\right), \ldots$ and $u_{s}=\frac{1}{2}\left(u_{s-1}+u_{k}\right),\{i, j$, 
$k, l\}=\{1,2,3,4\}$ and $u_{6}=u_{k}$, when $s=5$.

Proposition 2.3. $\left(\sigma, u_{0}\right)$ in (2) of Theorem 2.1 is one of the following.

(2-1) $u_{1} u_{2} u_{3} u_{4} u_{5} \cap N \subset\left\{u_{0}, u_{1}, u_{2}, u_{3}, u_{4}, u_{5}\right\}$.

$(2-2) \quad\left(u_{1} u_{2} u_{3} u_{4} u_{5} \cap N\right) \backslash\left\{u_{0}, u_{1}, u_{2}, u_{3}, u_{4}, u_{5}\right\}=\left\{u_{6}\right\}$ and $u_{0} \in \operatorname{Int}\left(u_{i} u_{6}\right)$, where $u_{6}=\frac{1}{2}\left(u_{j}+u_{k}\right)$ and $\{i, j, k\}=\{1,2,3\}$.

$(2-2-1) \quad u_{i}=\frac{1}{2}\left(u_{4}+u_{5}\right)$.

$(2-2-2) \quad \mathrm{u}_{6}=\frac{1}{2}\left(u_{4}+u_{5}\right)$.

$(2-2-3) \quad \tilde{u} \in \operatorname{Int}\left(u_{i} u_{6}\right)$ and hence $u_{0} \in u_{i} \tilde{u}$, where $\tilde{u}$ is the intersection point of $u_{1} u_{2} u_{3}$ and $u_{4} u_{5}$.

$(2-2-4) \quad u_{j}=\frac{1}{2}\left(u_{4}+u_{5}\right)$ and $u_{0}=\frac{1}{2}\left(u_{i}+u_{6}\right) \in N$.

$(2-3) \quad\left(u_{1} u_{2} u_{3} u_{4} u_{5} \cap N\right) \backslash\left\{u_{1}, u_{2}, u_{3}, u_{4}, u_{5}\right\}=\left\{u_{6}, \ldots, u_{s}\right\} \subset u_{i} u_{j} \quad(s \geqq 6), \quad u_{i}=$ $\frac{1}{2}\left(u_{4}+u_{5}\right), u_{0} \in u_{j} u_{k} u_{s}$ and $u_{0} \notin N$, where $u_{6}=\frac{1}{2}\left(u_{i}+u_{7}\right), \ldots$ and $u_{s}=\frac{1}{2}\left(u_{s-1}+\right.$ $\left.u_{j}\right)(s \geqq 7)$ or $u_{6}=\frac{1}{2}\left(u_{i}+u_{j}\right)(s=6)$.

(2-4) $\left(u_{1} u_{2} u_{3} u_{4} u_{5} \cap N\right) \backslash\left\{u_{0}, u_{1}, u_{2}, u_{3}, u_{4}, u_{5}\right\}=\left\{u_{6}, u_{7}, u_{8}\right\}, \quad u_{i}=\frac{1}{2}\left(u_{4}+u_{5}\right)$ and $u_{0}=\frac{1}{2}\left(u_{k}+u_{7}\right) \in N$, where $u_{6}=\frac{1}{3}\left(2 u_{i}+u_{j}\right), u_{7}=\frac{1}{3}\left(u_{i}+2 u_{j}\right)$ and $u_{8}=\frac{1}{2}\left(u_{i}+u_{k}\right)$.

Proof of Theorem 2.1. Let $\square=\{u \in \sigma \mid\langle v(\sigma), u\rangle=1\}$. Then $\sigma=\mathbb{R}_{\geqq 0} \square$ and $u_{0} \in \operatorname{Int}(\square)$. Moreover, $\partial \square$ has the natural polygonal decomposition $\{\tau \cap \square \mid \tau$ are faces of $\sigma$ with $\tau \neq\{0\}, \tau \neq \sigma\}$. Since the vertices of $\square$ belongs to $N$, we can take a triangulation $\Delta$ of $\partial \square$ so that $\Delta$ is a subdivision of the polygonal decomposition and that the set of vertices of $\Delta$ coincides with $\partial \square \cap N$. On the other hand, for each point $u$ on $\partial \square$, there exists the unique point $\hat{u}$ on $\partial \square$ with $u_{0} \in u \hat{u}$. We denote by $\lambda(u)$, the simplex of $\Delta$ with $\hat{u} \in \operatorname{Int}(\lambda(u))$.

(I) Assume that there exists an element $u_{1} \in \partial \square \cap N$ such that $\lambda\left(u_{1}\right)$ is a triangle of $\Delta$ and let $u_{2}, u_{3}$ and $u_{4}$ be the vertices of $\lambda\left(u_{1}\right)$. Then $u_{0} \in \operatorname{Int}\left(u_{1} u_{2} u_{3} u_{4}\right)$. Hence $\square=u_{1} u_{2} u_{3} u_{4}$, because $\left(\sigma, u_{0}\right)$ is minimal. When $u_{i} u_{j} \cap N=\left\{u_{i}, u_{j}\right\}$, for any $\{i, j\} \subset\{1,2,3,4\},\left(\sigma, u_{0}\right)$ is in the case of (1). Assume that $u_{i} u_{j} \cap N \neq\left\{u_{i}, u_{j}\right\}$, for a certain $\{i, j\} \subset\{1,2,3,4\}$. Then since $\left(\sigma, u_{0}\right)$ is minimal, we easily see that $u_{i} u_{j} \cap N=\left\{u_{i}, u_{j}, u_{5}\right\}$ and that $u_{0} \in \operatorname{Int}\left(u_{k} u_{l} u_{5}\right)$, where $u_{5}=\frac{1}{2}\left(u_{i}+u_{j}\right)$ and $\{i, j, k, l\}$ $=\{1,2,3,4\}$. Hence $\left(\sigma, u_{0}\right)$ is in the case of $(2)$.

(II) Assume that there exists an element $u_{1} \in \partial \square \cap N$ such that $\lambda\left(u_{1}\right)$ is an edge of $\Delta$ and let $u_{2}$ and $u_{3}$ be the vertices on $\lambda\left(u_{1}\right)$. Then $u_{0} \in \operatorname{Int}\left(u_{1} u_{2} u_{3}\right)$. Let $H=\mathbb{R} u_{1}+\mathbb{R} u_{2}+\mathbb{R} u_{3} \subset N_{\mathbb{R}}$. Then there exist certain elements $u_{4}$ and $u_{5}$ in $\partial \square \cap N$ such that $u_{4}, u_{5} \notin H$ and that $u_{4} u_{5} \cap H \neq \varnothing$, because $\square$ is the convex hull of $\partial \square \cap N, \operatorname{dim} \square=3$ and $u_{0} \in \operatorname{Int}(\square)$. Since $\left(\sigma, u_{0}\right)$ is minimal, $\square=u_{1} u_{2} u_{3} u_{4} u_{5}$. We denote by $\tilde{u}$, the intersection point of $u_{4} u_{5}$ and $H$. Then $\tilde{u}=a_{1} u_{1}+a_{2} u_{2}+$ $a_{3} u_{3}=b_{4} u_{4}+b_{5} u_{5}$, for certain real numbers $a_{1}, a_{2}, a_{3}$ and for certain positive real numbers $b_{4}, b_{5}$ with $a_{1}+a_{2}+a_{3}=b_{4}+b_{5}=1$. 
Lemma. One of the following holds.

(i) $a_{1}, a_{2}, a_{3} \geqq 0$.

(ii) $a_{i}<0, a_{j}>0, a_{k}>0$ and $u_{0} \in \operatorname{Int}\left(u_{i} \tilde{u}\right)$, where $\{i, j, k\}=\{1,2,3\}$.

(iii) $a_{i}<0, a_{j}>0$ and $a_{k}=0$, where $\{i, j, k\}=\{1,2,3\}$.

Proof. Suppose that $a_{i}, a_{j}<0$, where $\{i, j, k\}=\{1,2,3\}$. Then $a_{k}>0, u_{k}=$ $\left(1 / a_{k}\right) \tilde{u}+\left(-a_{i} / a_{k}\right) u_{i}+\left(-a_{j} / a_{k}\right) u_{j}$ and $1 / a_{k}+\left(-a_{i} / a_{k}\right)+\left(-a_{j} / a_{k}\right)=1$. Hence $u_{k} \in$ Int $(\sigma)$. It contradicts the fact that $u_{k} \in \partial \square \cap N$. Therefore, it suffices to show that $u_{0} \in \operatorname{Int}\left(u_{i} \tilde{u}\right)$, if $a_{i}<0, a_{j}>0, a_{k}>0$, where $\{i, j, k\}=\{1,2,3\}$. Since $u_{0} \in$ Int $\left(u_{1} u_{2} u_{3}\right)$, we have $u_{0}=c_{1} u_{1}+c_{2} u_{2}+c_{3} u_{3}$, for certain positive real numbers $c_{1}$, $c_{2}$ and $c_{3}$ with $c_{1}+c_{2}+c_{3}=1$. Suppose that $a_{j} / a_{k}<c_{j} / c_{k}$. Then $u_{0}=\left(\left(c_{i} a_{k}-\right.\right.$ $\left.\left.c_{k} a_{i}\right) / a_{k}\right) u_{i}+\left(\left(c_{j} a_{k}-c_{k} a_{j}\right) / a_{k}\right) u_{j}+\left(c_{k} / a_{k}\right) \tilde{u},\left(c_{i} a_{k}-c_{k} a_{i}\right)+\left(c_{j} a_{k}-c_{k} a_{j}\right)+c_{k}=$ $a_{k}, c_{j} a_{k}-c_{k} a_{j}>0, c_{i} a_{k}-c_{k} a_{i}>0$ and $c_{k}>0$. Hence $u_{0} \in \operatorname{Int}\left(u_{i} u_{j} u_{4} u_{5}\right)$ and $u_{k} \notin$ $u_{i} u_{j} u_{4} u_{5}$. It contradicts the assumption that $\left(\sigma, u_{0}\right) \in \widetilde{\mathscr{E}}_{0}^{3}$. Therefore, $a_{j} / a_{k}=c_{j} / c_{k}$. Thus we have $u_{0}=\left(\left(c_{i} a_{j}-c_{j} a_{i}\right) / a_{j}\right) u_{\mathrm{i}}+\left(c_{j} / a_{j}\right) \tilde{u} \in \operatorname{Int}\left(u_{i} \tilde{u}\right)$.

q.e.d.

Proof of Theorem 2.1 continued. When (i) in the above lemma holds, $\tilde{u} \in u_{1} u_{2} u_{3}$. Hence $\left(\sigma, u_{0}\right)$ is in the case of (2). When (ii) in the above lemma holds, $u_{0} \in \operatorname{Int}\left(u_{i} u_{4} u_{5}\right)$ and $u_{j} u_{k} \cap u_{i} u_{4} u_{5} \neq \varnothing$, because $\left(1 /\left(a_{J}+a_{k}\right)\right)\left(a_{j} u_{j}+a_{k} u_{k}\right)$ $=\left(1 /\left(b_{4}+b_{5}-a_{i}\right)\right)\left(b_{4} u_{4}+b_{5} u_{5}-a_{i} u_{i}\right)$. Hence $\left(\sigma, u_{0}\right)$ is in the case of (2). When (iii) in the above lemma holds, $\tilde{u}=a_{i} u_{i}+a_{j} u_{j}$. Then $u_{j}=\left(1 / a_{j}\right) \tilde{u}+\left(-a_{i} / a_{j}\right) u_{i} \in$ $\tilde{u} u_{i}$. Hence $\square=u_{i} u_{k} u_{4} u_{5}$. We already considered this case in (I).

(III) Assume that $\hat{u} \in N$, for all $u \in \partial \square \cap N$. There exist certain elements $u_{1}$, $u_{3}$ and $u_{5} \in \partial \square \cap N$ such that $u_{1}-u_{0}, u_{3}-u_{0}$ and $u_{5}-u_{0}$ are linearly independent, because $\operatorname{dim} \square=3$. Let $u_{2}=\hat{u}_{1}$, let $u_{4}=\hat{u}_{3}$ and let $u_{6}=\hat{u}_{5}$. Then $u_{0} \in$ $\operatorname{Int}\left(u_{1} u_{2}\right)$, Int $\left(u_{3} u_{4}\right)$, Int $\left(u_{5} u_{6}\right)$ and hence $u_{0} \in \operatorname{Int}\left(u_{1} u_{2} \ldots u_{6}\right)$. Therefore, $\square=u_{1} u_{2} \ldots u_{6}$ and $\square \cap N \subset\left\{u_{0}, u_{1}, \ldots, u_{6}\right\}$, because $\left(\sigma, u_{0}\right)$ is minimal. Then $\left(\sigma, u_{0}\right)$ is in the case of (3).

q.e.d.

Proof of Proposition 2.2. We may only consider the case that $\left(u_{1} u_{2} u_{3} u_{4} \cap\right.$ $N) \backslash\left\{u_{0}, u_{1}, u_{2}, u_{3}, u_{4}\right\} \neq \varnothing$.

(I) Assume that there exists an element $u \in \operatorname{Int}\left(u_{1} u_{2} u_{3}\right) \cap N$ with $u \neq \hat{u}_{4}$, where $\hat{u}_{4}$ is the point in $\partial\left(u_{1} u_{2} u_{3} u_{4}\right)$ with $u_{0} \in \operatorname{Int}\left(u_{4} \hat{u}_{4}\right)$. Then $\hat{u}_{4} \in u_{1} u, u_{2} u$ or $u_{3} u$, because $\left(\sigma, u_{0}\right)$ is minimal. Hence we may assume that $\hat{u}_{4} \in u_{3} u$. Let $H=$ $\mathbb{R} u+\boldsymbol{R} u_{3}+\mathbb{R} u_{4}$. Then $\left(u_{1} u_{2} u_{3} u_{4} \cap N\right) \backslash\left\{u_{1}, u_{2}\right\} \subset H$, because $u_{0} \in \operatorname{Int}\left(u u_{3} u_{4}\right)$. On the other hand, $u_{1} u_{2}$ intersect $H$ at a point, which we denote by $\tilde{u}$. Then $u_{1} u_{2} u_{3} \cap H=\tilde{u} u_{3}$. We may assume that $\tilde{u} u_{3} \cap N=\left\{u_{3}, u_{5}, \ldots, u_{s}\right\}$, where $u_{s}=$ $\frac{1}{2}\left(u_{3}+u_{s-1}\right), u_{s-1}=\frac{1}{2}\left(u_{s}+u_{s-2}\right), \ldots$ and $u_{6}=\frac{1}{2}\left(u_{7}+u_{5}\right)$. Then $u_{0} \in u_{3} u_{4} u_{s}$. Otherwise, $u_{0} \in \operatorname{Int}\left(u_{1} u_{2} u_{4} u_{s}\right)$. Moreover, $u_{5}=\frac{1}{3}\left(u_{1}+u_{2}+u_{6}\right)$, because $u_{5} \in \operatorname{Int}\left(u_{1} u_{2} u_{6}\right)$ and $u_{1} u_{2} u_{6} \cap N=\left\{u_{1}, u_{2}, u_{5}, u_{6}\right\}$. Hence $\tilde{u}=\frac{1}{2}\left(u_{1}+u_{2}\right)$. In the following, we show that $\tilde{u} u_{4} \cap N=\left\{u_{4}\right\}$, i.e., $\partial\left(u_{1} u_{2} u_{3} u_{4}\right) \cap N=\left\{u_{1}, u_{2}, u_{3}, u_{4}, \ldots, u_{s}\right\}$.

(I-i) Assume that $u_{0} \notin N$. Since $u_{3} u_{4} u_{s} \cap N=\left\{u_{3}, u_{4}, u_{s}\right\},\left\{u_{4}-u_{3}, u_{s}-u_{3}\right\}$ 
is a basis of $\left(\boldsymbol{R}\left(u_{4}-u_{3}\right)+\boldsymbol{R}\left(u_{s}-u_{3}\right)\right) \cap N$. On the other hand, any point $u^{\prime}$ on $\tilde{u} u_{4}$ is expressed as $u^{\prime}=a u_{4}+b \tilde{u}$, for certain non-negative real numbers $a$ and $b$ with $a+b=1$. Hence $u^{\prime}-u_{3}=a\left(u_{4}-u_{3}\right)+b\left(\tilde{u}-u_{3}\right)=a\left(u_{4}-u_{3}\right)+b((s-4+$ $\left.\left.\frac{1}{2}\right)\left(u_{s}-u_{3}\right)\right)$. Therefore, $\tilde{u} u_{4} \cap N=\left\{u_{4}\right\}$.

(I-ii) Assume that $u_{0} \in N$. If $u_{0} \in u_{4} u_{s}$ (resp. $u_{0} \in \operatorname{Int}\left(u_{3} u_{4} u_{s}\right)$ ), then $u_{0}=$ $\frac{1}{2}\left(u_{4}+u_{s}\right)$ (resp. $u_{0}=\frac{1}{3}\left(u_{3}+u_{4}+u_{s}\right)$ ). Hence $s \leqq 6$ (resp. $\left.s=5\right)$. Otherwise, $\frac{1}{2}\left(u_{4}+u_{s-2}\right)=\frac{1}{2}\left(2 u_{0}-u_{s}+3 u_{s}-2 u_{3}\right)=u_{0}+u_{s}-u_{3} \in N \cap \operatorname{Int}(\sigma) \quad\left(\right.$ resp. $\frac{1}{3}\left(2 u_{4}+\right.$ $\left.\left.u_{s-1}\right)=\frac{1}{3}\left(2\left(3 u_{0}-u_{3}-u_{s}\right)+2 u_{s}-u_{3}\right)=2 u_{0}-u_{3} \in N \cap \operatorname{Int}(\sigma)\right)$. Then we easily see that $\tilde{u} u_{4} \cap N=\left\{u_{4}\right\}$.

(II) Assume that $u_{1} u_{2} u_{3} u_{4} \cap N \subset\left\{u_{0}, u_{1}, u_{2}, u_{3}, u_{4}, \hat{u}_{1}, \hat{u}_{2}, \hat{u}_{3}, \hat{u}_{4}\right\}$ and that $\hat{u}_{4} \in$ $N$. Then $\hat{u}_{4}=\frac{1}{3}\left(u_{1}+u_{2}+u_{3}\right)$. Suppose that $\hat{u}_{3} \in N$. Then $\hat{u}_{3}=\frac{1}{3}\left(u_{1}+u_{2}+u_{4}\right)$. Hence $\quad \hat{u}_{4}-\hat{u}_{3}=\frac{1}{3}\left(u_{3}-u_{4}\right)$. Then $u_{3}+\hat{u}_{3}-\hat{u}_{4}=u_{3}+\frac{1}{3}\left(u_{4}-u_{3}\right)=\frac{2}{3} u_{3}+\frac{1}{3} u_{4} \in$ Int $\left(u_{3} u_{4}\right) \cap N$. This contradicts the assumption that $u_{3} u_{4} \cap N=\left\{u_{3}, u_{4}\right\}$. Therefore, $u_{1} u_{2} u_{3} u_{4} \cap N \subset\left\{u_{0}, u_{1}, u_{2}, u_{3}, u_{4}, \hat{u}_{4}\right\}$.

q.e.d.

Proof of Proposition 2.3. We easily see that $\left(u_{1} u_{2} \ldots u_{5} \cap N\right) \backslash\left\{u_{4}, u_{5}\right\}=$ $u_{1} u_{2} u_{3} \cap N$, because $\left(\sigma, u_{0}\right)$ is minimal and $u_{0} \in \operatorname{Int}\left(u_{1} u_{2} u_{3}\right)$. We may only consider the case that $\partial\left(u_{1} u_{2} u_{3}\right) \cap N \neq\left\{u_{1}, u_{2}, u_{3}\right\}$. Let $\tilde{u}$ be the intersection point of $u_{1} u_{2} u_{3}$ and $u_{4} u_{5}$.

(I) Assume that $\tilde{u}=u_{1}$.

(I-i) If $u_{2} u_{3} \cap N \neq\left\{u_{2}, u_{3}\right\}$, then $u_{2} u_{3} \cap N=\left\{u_{2}, u_{3}, u_{6}\right\}$ and $u_{0} \in u_{1} u_{6}$, where $u_{6}=\frac{1}{2}\left(u_{2}+u_{3}\right)$. Hence $\partial u_{1} u_{2} u_{3} \cap N=\left\{u_{1}, u_{2}, u_{3}, u_{6}\right\}$, because $\left(\sigma, u_{0}\right)$ is minimal. Then $\left(\sigma, u_{0}\right)$ is in the case of $(2-2-1)$.

(I-ii) If $u_{2} u_{3} \cap N=\left\{u_{2}, u_{3}\right\}$, then $\operatorname{Int}\left(u_{1} u_{2}\right) \cap N \neq \varnothing$ or $\operatorname{Int}\left(u_{1} u_{3}\right) \cap N \neq \varnothing$. Hence we may assume that $\operatorname{Int}\left(u_{1} u_{2}\right) \cap N=\left\{u_{6}, u_{7}, \ldots, u_{s}\right\}(s \geqq 6)$, where $u_{6}=$ $\frac{1}{2}\left(u_{1}+u_{7}\right)=\frac{1}{2}\left(u_{6}+u_{8}\right), \ldots, u_{s}=\frac{1}{2}\left(u_{s-1}+u_{2}\right)$. Then $u_{0} \in u_{s} u_{2} u_{3}$, because $\left(\sigma, u_{0}\right)$ is minimal.

(I-ii-a) When $u_{0} \notin N, u_{2} u_{3} u_{s} \cap N=\left\{u_{2}, u_{3}, u_{s}\right\}$. Hence $\left\{u_{3}-u_{2}, u_{s}-u_{2}\right\}$ is a basis of $\left(\mathbb{R}\left(u_{3}-u_{2}\right)+\mathbb{R}\left(u_{s}-u_{2}\right)\right) \cap N$. On the other hand, any point $u^{\prime}$ on $u_{1} u_{3}$ is expressed as $u^{\prime}=a u_{1}+b u_{3}$ for certain non-negative real numbers $a$ and $b$ with $a+b=1$. Hence $u^{\prime}-u_{2}=a\left(u_{1}-u_{2}\right)+b\left(u_{3}-u_{2}\right)=a(s-4) \quad\left(u_{s}-u_{2}\right)+b\left(u_{3}-\right.$ $\left.u_{2}\right)$. Therefore, $u_{1} u_{3} \cap N=\left\{u_{1}, u_{3}\right\}$. Then $\left(\sigma, u_{0}\right)$ is in the case of $(2-3)$.

(I-ii-b) When $u_{0} \in N$ and $u_{0} \in u_{3} u_{s}$, we have $s=6$ or 7 . Otherwise $\frac{1}{2}\left(u_{s-2}+\right.$ $\left.u_{3}\right)=\frac{1}{2}\left(2 u_{s-1}-u_{s}+2 u_{0}-u_{s}\right)=u_{0}+u_{s-1}-u_{s} \in N \cap \operatorname{Int}(\sigma)$. If $s=6$, then $u_{6}=$ $\frac{1}{2}\left(u_{1}+u_{2}\right)$ and $u_{0}=\frac{1}{2}\left(u_{6}+u_{3}\right)$. Then $\left(\sigma, u_{0}\right)$ is in the case of $(2-2-4)$. If $s=7$, then $u_{6}=\frac{1}{2}\left(u_{1}+u_{7}\right), u_{7}=\frac{1}{2}\left(u_{6}+u_{2}\right), u_{0}=\frac{1}{2}\left(u_{7}+u_{3}\right)$ and $u_{8}:=\frac{1}{2}\left(u_{1}+u_{3}\right)=\frac{1}{2}\left(2 u_{6}-\right.$ $\left.u_{7}+2 u_{0}-u_{7}\right)=u_{0}+u_{6}-u_{7} \in N$. Then $\left(\sigma, u_{0}\right)$ is in the case of $(2-4)$.

(I-ii-c) When $u_{0} \in N$ and $u_{0} \in \operatorname{Int}\left(u_{2} u_{3} u_{s}\right)$, we have $u_{0}=\frac{1}{3}\left(u_{2}+u_{3}+u_{s}\right)$ and $s=6$. Otherwise $\frac{1}{3}\left(2 u_{3}+u_{s-1}\right)=\frac{1}{3}\left(2\left(3 u_{0}-u_{2}-u_{s}\right)+\left(2 u_{s}-u_{2}\right)\right)=2 u_{0}-u_{2} \in$ $N \cap \operatorname{Int}(\sigma)$. Hence $u_{6}=\frac{1}{2}\left(u_{1}+u_{2}\right), \quad u_{7}:=\frac{1}{3}\left(2 u_{3}+u_{1}\right)=2 u_{0}-u_{2}, \quad u_{8}:=\frac{1}{3}\left(u_{3}+\right.$ $\left.2 u_{1}\right)=u_{0}+u_{6}-u_{2} \in N$ and $u_{0}=\frac{1}{2}\left(u_{2}+u_{7}\right)$. Then $\left(\sigma, u_{0}\right)$ is in the case of $(2-4)$. (II) Assume that $\tilde{u} \in \operatorname{Int}\left(u_{2} u_{3}\right)$. Then $u_{0} \in u_{1} \tilde{u}$. Otherwise, $u_{0} \in \operatorname{Int}\left(u_{1} u_{2} u_{4} u_{5}\right)$ 
or Int $\left(u_{1} u_{3} u_{4} u_{5}\right)$. Moreover, $\left(u_{1} u_{2} u_{3} \cap N\right) \backslash\left\{u_{0}, u_{1}, u_{2}, u_{3}\right\}=\{\tilde{u}\}$, because $\left(\sigma, u_{0}\right)$ is minimal. Therefore, $\tilde{u}=\frac{1}{2}\left(u_{2}+u_{3}\right)=\frac{1}{2}\left(u_{4}+u_{5}\right) \in N$. Then $\left(\sigma, u_{0}\right)$ is in the case of $(2-2-2)$.

(III) Assume that $\tilde{u} \in \operatorname{Int}\left(u_{1} u_{2} u_{3}\right)$. If there exists a lattice point $u_{6}$ on $\operatorname{Int}\left(u_{2} u_{3}\right)$, then $u_{0} \in u_{6} u_{1}$ and hence $\tilde{u} \in u_{6} u_{1}$. Otherwise, $u_{1} u_{2} u_{4} u_{5} u_{6}$ or $u_{1} u_{3} u_{4} u_{5} u_{6}$ contains $u_{0}$ in the interior and is strictly.contained in $u_{1} u_{2} u_{3} u_{4} u_{5}$. Moreover, we see that $u_{0} \in u_{1} \tilde{u}$ and $u_{1} u_{2} u_{3} \cap N \subset\left\{u_{0}, u_{1}, u_{2}, u_{3}, u_{6}\right\}$, because $\left(\sigma, u_{0}\right)$ is minimal. Then $\left(\sigma, u_{0}\right)$ is in the case of $(2-2-3)$

q.e.d.

\section{§3. Representatives of the pairs in $\mathscr{E}_{0}^{3}$}

In this section, we need the following lemmas, which are easily obtained from the terminal lemma [4] (see also [9, Lemma 3.6]).

Lemma 3.1. Assume that $u_{1}, u_{2}, u_{3}$ and $u_{4}$ are elements in $N$ with $u_{1} u_{2} u_{3} u_{4}$ $\cap N=\left\{u_{1}, u_{2}, u_{3}, u_{4}\right\}$. Moreover, assume that $u_{1}, u_{2}, u_{3}$ and $u_{4}$ are linearly independent and that there exists an element $v_{0}$ in $N^{*}$ such that $\left\langle v_{0}, u_{i}\right\rangle=1$. for $i=1$ through 4. Then there exists an element $g$ in $G L(N)$ such that $g\left(u_{1}\right)=(0,0,0,1)$, that $g\left(u_{2}\right)=(1,0,0,1)$, that $g\left(u_{3}\right)=(0,1,0,1)$ and that $g\left(u_{4}\right)=(o, p, q, 1)$, where $o, p, q \in \mathbb{Z}, 0 \leqq o, p<q$, g.c.d. $(o, q)=$ g.c.d. $(p, q)=1$ and $o=1, p=1$ or $o+p=q$.

Lemma 3.2. Under the same assumptions as in Lemma 3.1, there exists an element $g$ in $G L(N)$ such that $\mathrm{g}\left(u_{i}\right)=(0,0,0,1)$, that $g\left(u_{j}\right)=(1,0,0,1)$, that $g\left(u_{k}\right)=$ $(0,1,0,1)$ and that $g\left(u_{l}\right)=(1, p, q, 1)$, where $\{i, j, k, l\}=\{1,2,3,4\}, 0 \leqq p \leqq \frac{q}{2}$ and g.c.d. $(p, q)=1$. Moreover, we may assume that $i=1$ or $l=4$.

Assume that $\sigma=\mathbb{R}_{\geqq_{0}} u_{1}+\boldsymbol{R}_{\geqq 0} u_{2}+\ldots+\mathbb{R}_{\geqq 0} u_{t}$ is a 4-dimensional strongly convex rational cone in $N_{R}$ satisfying the condition $(\mathrm{G})$. Moreover, assume that $\mathbb{R}_{\geqq 0} u_{t}$ is a 1 -dimensional face of $\sigma$, for $i=1$ through $t$.

Proposition 3.3. Assume that $t=4$, that $\{u \in \sigma \cap N \mid\langle v(\sigma), u\rangle=1\}=\left\{u_{1}, u_{2}\right.$, $\left.u_{3}, u_{4}\right\}$ and that $W_{\sigma}(v(\sigma)) \neq \varnothing$. Then there exists an element $g$ in $G L(N)$ such that $g\left(u_{i}\right)=(0,0,0,1)$, that $g\left(u_{\jmath}\right)=(1,0,0,1)$, that $g\left(u_{k}\right)=(0,1,0,1)$ and that $g\left(u_{l}\right)=$ $(1, p, q, 1)$, where $\{i, j, k, l\}=\{1,2,3,4\}$ and $(p, q)=(0,1),(1,2),(1,3),(1,4),(2,5)$, $(2,7),(3,7),(3,8)$ or $(3,10)$.

Proof. By Lemma 3.2, there exists an element $g$ in $G L(N)$ such that $g\left(u_{i}\right)=(0,0,0,1)$, that $g\left(u_{j}\right)=(1,0,0,1)$, that $g\left(u_{k}\right)=(0,1,0,1)$ and that $g\left(u_{l}\right)=$ $(1, p, q, 1)$, where $\{i, j, k, l\}=\{1,2,3,4\}, p, q \in \mathbb{Z}, 0 \leqq p \leqq q / 2$ and g.c.d. $(p, q)=1$. We may only consider the case that $i=1, j=2, k=3$ and $l=4$. When $q \leqq 4$, $(p, q)=(0,1),(1,2),(1,3)$ or $(1,4)$. In these cases, we easily see that $W_{\sigma}(v(\sigma)) \neq \varnothing$. In the following, we consider the case that $q>4$. Then $0<p<\frac{q}{2}$. Let $v_{1}=$ 
$\frac{1}{q}(0,0,1,0)$, let $v_{2}=\frac{1}{q}(0, q,-p, 0)$, let $v_{3}=\frac{1}{q}(q, 0,-1,0)$, let $v_{4}=\frac{1}{q}(-q,-q, p, q)$ and let $u_{0}=\frac{1}{4}(2, p+1, q, 4)$. Then $(g \sigma)^{*}=\boldsymbol{R}_{\geqq 0} v_{1}+\boldsymbol{R}_{\geqq 0} v_{2}+\boldsymbol{R}_{\geqq 0} v_{3}+\boldsymbol{R}_{\geqq 0} v_{4}, \quad u_{0} \in$ Int $(g \sigma), \quad\left\langle v_{1}, u_{0}\right\rangle=\left\langle v_{2}, u_{0}\right\rangle=\left\langle v_{3}, u_{0}\right\rangle=\left\langle v_{4}, u_{0}\right\rangle=\frac{1}{4} \quad$ and $\left\langle v_{0}, u_{0}\right\rangle=1$, where $v_{0}=g(v(\sigma))(=(0,0,0,1))$.

Step 1. First, we examine when $u_{0} \in W_{g \sigma}\left(v_{0}\right)=g\left(W_{\sigma}(v(\sigma))\right)$. Assume that $v:=a v_{1}+b v_{2}+c v_{3}+d v_{4} \in N^{*}$. Then $c-d, b-d,(a-b p-c+d p) / q, d \in \mathbb{Z}$. Hence $a, b, c, d \in Z$ and $a-b p-c+d p \equiv 0(\bmod q)$. By an easy calculation, we obtain:

Lemma 3.3.1. (0) $L_{0}:=\left\{v_{1}+v_{2}+v_{3}+v_{4}, 2 v_{1}+2 v_{3}, 2 v_{2}+2 v_{4}\right\} \subset N^{*}$.

(1) For $v \in L_{1}:=\left\{2 v_{1}+2 v_{2}, 2 v_{3}+2 v_{4}, v_{1}+2 v_{2}+v_{4}, 2 v_{1}+v_{2}+v_{3}, v_{1}+2 v_{3}+\right.$ $\left.v_{4}, v_{2}+v_{3}+2 v_{4}\right\}$,

$v \in N^{*}$ if and only if $p=1$.

(2) For $v \in L_{2}:=\left\{v_{1}+3 v_{2}, v_{3}+3 v_{4}\right\}$,

$v \in \mathrm{N}^{*}$ if and only if $3 p-1=q$.

(3) For $v \in L_{3}:=\left\{3 v_{1}+v_{2}, 3 v_{3}+v_{4}\right\}$,

$v \in N^{*}$ if and only if $p=3$.

(4) For $v \in L_{4}:=\left\{3 v_{1}+v_{4}, v_{2}+3 v_{3}\right\}$,

$v \in N^{*}$ if and only if $p+3=q$.

(5) For $v \in L_{5}:=\left\{2 v_{1}+2 v_{4}, 2 v_{2}+2 v_{3}\right\}$,

$v \in N^{*}$ if and only if $2 p+2=q$.

(6) For $v \in L_{6}:=\left\{v_{1}+3 v_{4}, 3 v_{2}+v_{3}\right\}$,

$v \in N^{*}$ if and only if $3 p+1=q$.

$\left\{v \in(g \sigma)^{*} \cap N^{*} \mid\left\langle v, u_{0}\right\rangle=1\right\} \subset L_{0} \cup L_{1} \cup \ldots \cup L_{6}$. Moreover, $L_{0} \cup L_{i}$ is contained in a sublinear space of $N_{R}^{*}$, for each $1 \leqq i \leqq 6$.

If $u_{0} \in W_{g \sigma}\left(v_{0}\right)$, then at least two conditions in (1) (6) of the above lemma hold at the same time. When $(p, q)=(2,5)$, the conditions in (2) and (4) hold. When $(p, q)=(3,8)$, the conditions in (2), (3) and (5) hold. When $(p, q)=(3,10)$, the conditions in (3) and (6) hold. In fact, in these cases, $u_{0} \in W_{g \sigma}\left(v_{0}\right)$. For all pairs $(p, q)$ with g.c.d. $(p, q)=1$ except the above ones, any two conditions in (1) (6) do not hold at the same time.

Step 2. Next, we examine when $W_{g \sigma}\left(v_{0}\right) \backslash\left\{u_{0}\right\} \neq \varnothing$. Then there should exist an element $v \in(g \sigma)^{*} \cap N^{*}$ such that $\left\langle v, u_{0}\right\rangle<1$ and that $v_{0}$ and $v$ are linearly independent, as we see in the proof of Theorem 1.7. We easily obtain:

Lemma 3.3.2. (0) $L_{0}^{\prime}:=\left\{v_{1}+v_{3}, v_{2}+v_{4}\right\} \subset N^{*}$.

(1) For $v \in L_{1}^{\prime}:=\left\{v_{1}+v_{2}, v_{3}+v_{4}\right\}$,

$v \in N^{*}$ if and only if $p=1$.

(2) For $v \in L_{2}^{\prime}:=\left\{2 v_{1}+v_{2}, 2 v_{3}+v_{4}\right\}$,

$v \in N^{*}$ if and only if $p=2$.

(3) For $v \in L_{3}^{\prime}:=\left\{v_{1}+2 v_{4}, 2 v_{2}+v_{3}\right\}$, 
$v \in N^{*}$ if and only if $2 p+1=q$.

$\left\{v \in(g \sigma)^{*} \cap N^{*} \mid 0<\left\langle v, u_{0}\right\rangle<1\right\} \subset L_{0}^{\prime} \cup L_{1}^{\prime} \cup L_{2}^{\prime} \cup L_{3}^{\prime}$.

If $\left\langle v_{0}, u\right\rangle=\left\langle v_{i}+v_{j}, u\right\rangle=1$, for an element $u$ in $N_{R}$, then $\left\langle v_{k}+v_{l}, u\right\rangle=0$ and hence $u \notin \operatorname{Int}(g \sigma)$, because $v_{0}=v_{1}+v_{2}+v_{3}+v_{4}$, where $\{i, j, k, l\}=\{1,2,3,4\}$. Therefore, if $u \in W_{g \sigma}\left(v_{0}\right) \backslash\left\{u_{0}\right\}$, then $\langle v, u\rangle=1$, for an element $v$ in $\left(L_{2}^{\prime} \cup L_{3}^{\prime}\right)$ $\cap N^{*}$ and hence $p=2$ or $2 p+1=q$.

Step 2-i. Assume that $p=2$ and that there exists an element $u$ in $W_{g \sigma}\left(v_{0}\right)$ with $\left\langle 2 v_{3}+v_{4}, u\right\rangle=1$. Let $u_{1}=\frac{1}{6}(3,3, q, 6)$. Then $u_{1} \in \operatorname{Int}(g \sigma),\left\langle v_{1}, u_{1}\right\rangle=\left\langle v_{2}, u_{1}\right\rangle$ $=\frac{1}{6}, \quad\left\langle v_{3}, u_{1}\right\rangle=\left\langle v_{4}, \mathrm{u}_{1}\right\rangle=\frac{1}{3} \quad$ and hence $\left\langle v_{0}, u_{1}\right\rangle=\left\langle 2 v_{3}+v_{4}, u_{1}\right\rangle=1$. Since $\left\langle v_{0},(0,0,1,0)\right\rangle=0$, we see that $u_{1} \notin W_{g \sigma}\left(v_{0}\right)$, by the following lemma, which and the next lemma we obtain by an easy calculation.

Lemma 3.3.3. $\left\{v \in(g \sigma)^{*} \cap N^{*} \mid\left\langle v, u_{1}\right\rangle=1\right\} \subset\left\{v \in N_{\boldsymbol{R}}^{*} \mid\langle v,(0,0,1,0)\rangle \leqq 0\right\}$.

Lemma 3.3.4. (0) $M_{0}:=\left\{v_{2}+v_{4}, v_{1}+v_{3}, 2 v_{1}+v_{2}\right\} \subset N^{*}$.

(1) For $v \in M_{1}:=\left\{2 v_{2}+v_{3}, v_{1}+2 v_{4}, v_{1}+3 v_{2}, 3 v_{1}+v_{4}, 5 v_{1}, 5 v_{2}\right\}, v \in N^{*}$ if and only if $q=5$.

(2) For $v \in M_{2}:=\left\{v_{1}+4 v_{2}, 3 v_{2}+v_{3}\right\}$,

$v \in N^{*}$ if and only if $q=7$.

$\left\{v \in(g \sigma)^{*} \cap N^{*} \mid 0<\left\langle v, u_{1}\right\rangle<1\right\} \subset M_{0} \cup M_{1} \cup M_{2}$.

Assume that $\left\langle 2 v_{1}+v_{2}, u\right\rangle=\left\langle 2 v_{3}+v_{4}, u\right\rangle=\left\langle v_{0}, u\right\rangle=1$, for an element $u$ in $N_{R}$. Then $\left\langle v_{2}+v_{4}, u\right\rangle=0$. Hence $u \notin \operatorname{Int}(g \sigma)$. Therefore, $q=5$ or $q=7$, by the above lemma.

Step 2-ii. Assume that $p=2$ and that there exists an element $u$ in $W_{g \sigma}\left(v_{0}\right)$ such that $\left\langle 2 v_{1}+v_{2}, u\right\rangle=1$. Then we obtain the same results as in Step $2-\mathrm{i}$, by the same way, letting $u_{1}=\frac{1}{6}(3,6,2 q, 6)$.

Step 2-iii. Assume that $q=2 p+1$ and that there exists an element $u$ in $W_{g \sigma}\left(v_{0}\right)$ with $\left\langle 2 v_{2}+v_{3}, u\right\rangle=1$. Let $u_{1}=\frac{1}{6}(3, p+2, q, 6)$. Then $u_{1} \in \operatorname{Int}(g \sigma)$ and $\left\langle v_{0}, u_{1}\right\rangle=\left\langle 2 v_{2}+v_{3}, u_{1}\right\rangle=1$. Since $\left\langle v_{0},(0,1,2,0)\right\rangle=0$, we see that $u_{1} \notin W_{g \sigma}\left(v_{0}\right)$, by the following lemma, which and the next lemma we obtain by an easy calculation.

Lemma 3.3.5. $\left\{v \in(g \sigma)^{*} \cap N^{*} \mid\left\langle v, u_{1}\right\rangle=1\right\} \subset\left\{v \in N_{R}^{*} \mid\langle v,(0,1,2,0)\rangle \geqq 0\right\}$.

Lemma 3.3.6. (0) $K_{0}:=\left\{v_{2}+v_{4}, v_{1}+2 v_{4}, v_{1}+v_{3}\right\} \subset N^{*}$.

(1) For $v \in K_{1}:=\left\{5 v_{4}, v_{3}+3 v_{4}, 2 v_{3}+v_{4}, 2 v_{1}+v_{2}, 3 v_{1}+v_{4}, 5 v_{1}\right\}, v \in N^{*}$ if and only if $p=2, q=5$.

(2) For $v \in K_{2}:=\left\{3 v_{1}+v_{2}, 4 v_{1}+v_{4}\right\}$,

$v \in \mathrm{N}^{*}$ if and only if $p=3, q=7$.

$\left\{v \in(g \sigma)^{*} \cap N^{*} \mid 0<\left\langle v, u_{1}\right\rangle<1\right\} \subset K_{0} \cup K_{1} \cup K_{2}$. 
Assume that $\left\langle v_{1}+2 v_{4}, u\right\rangle=\left\langle 2 v_{2}+v_{3}, u\right\rangle=\left\langle v_{0}, u\right\rangle=1$, for an element $u$ in $N_{R}$. Then $\left\langle v_{1}+v_{3}, u\right\rangle=0$. Hence $u \notin \operatorname{Int}(g \sigma)$. Therefore, $(p, q)=(2,5)$ or $(3,7)$, by the above lemma.

Step 2-iv. Assume that $q=2 p+1$ and that there exists an element $u$ in $W_{\mathrm{g} \sigma}\left(v_{0}\right)$ such that $\left\langle v_{1}+2 v_{4}, u\right\rangle=1$. Then we obtain the same results as in Step 2 -iii, by the same way, letting $u_{1}=\frac{1}{6}(3, q, 2 q, 6)$.

q.e.d.

Proposition 3.4. Assume that $t=4$ and that $\{u \in \sigma \cap N \mid\langle v(\sigma), u\rangle=$ $1\} \backslash\left\{u_{1}, u_{2}, u_{3}, u_{4}\right\}$ consists of only one point $u_{0}$ which is in Int $(\sigma)$. Then there exists an element $g$ in $G L(N)$ such that $g\left(u_{i}\right)=(1,0,0,1)$, that $g\left(u_{j}\right)=(0,1,0,1)$ and that $\left(g\left(u_{k}\right), g\left(u_{l}\right)\right)=((0,0,1,1),(-1,-1,-1,1)), \quad((1,1,2,1),(-1,-1,-1.1))$, $((1,1,3,1),(-1,-1,-2,1)),((1,2,5,1),(-1,-1,-1,1)),((1,2,5,1),(-1,-1,-2,1))$, $((1,2,5,1),(-2,-3,-5,1)),((1,2,7,1),(-1,-1,-2,1))$ or $((1,3,7,1),(-1,-2,-3,1))$, where $\{i, j, k, l\}=\{1,2,3,4\}$.

Proof. We may only consider the case that $\left|\operatorname{det}\left(u_{0}, u_{1}, u_{2}, u_{3}\right)\right| \geqq \mid \operatorname{det}$ $\left(u_{0}, u_{1}, u_{2}, u_{4}\right)|,| \operatorname{det}\left(u_{0}, u_{1}, u_{3}, u_{4}\right)|,| \operatorname{det}\left(u_{0}, u_{2}, u_{3}, u_{4}\right) \mid$. Since $u_{0} u_{1} u_{2} u_{3} \cap N=\left\{u_{0}\right.$, $\left.u_{1}, u_{2}, u_{3}\right\}$, by Lemma 3.2 , there exists an element $g$ in GL(N) such that $g\left(u_{0}\right)=$ $(0,0,0,1)$, that $g\left(u_{\mathrm{i}}\right)=(1,0,0,1)$, that $g\left(u_{j}\right)=(0,1,0,1)$ and that $g\left(u_{\mathrm{k}}\right)=(1, p, q, 1)$, where $p, q \in \mathbb{Z}, q>0,0 \leqq p \leqq \frac{q}{2}$ and g.c.d. $(p, q)=1$. Here, we may only consider the case that $i=1, j=2$ and $k=3$. Since $u_{0} \in \operatorname{Int}\left(u_{1} u_{2} u_{3} u_{4}\right)$ and $\sigma$ satisfies the condition $(G), g\left(u_{4}\right)=(-s,-t,-u, 1)$, for certain positive integers $s, t$ and $u$. Let $\tilde{u}=\frac{1}{q+u}\left(q g\left(u_{4}\right)+u g\left(u_{3}\right)\right)=\frac{1}{q+u}(u-s q, p u-t q, 0, q+u) . \quad$ Since $\quad g\left(u_{0}\right) \in$ Int $\left(\tilde{u} g\left(u_{1}\right) g\left(u_{2}\right)\right)$, we have $u-s q<0, p u-t q<0$. On the other hand, we have $\left|\operatorname{det}\left(u_{0}, u_{1}, u_{2}, u_{4}\right)\right|=u \leqq q,\left|\operatorname{det}\left(u_{0}, u_{2}, u_{3}, u_{4}\right)\right|=|s q-u| \leqq q$ and $\mid \operatorname{det}\left(u_{0}\right.$, $\left.u_{1}, u_{3}, u_{4}\right)|=| p u-t q \mid \leqq q$. Thus we obtain the following inequalities.

(1) $1 \leqq u \leqq q, \frac{u}{q}<s \leqq \frac{u}{q}+1, p \frac{u}{q}<t \leqq p \frac{u}{q}+1$.

When $\mathrm{q}=1$, we have $p=0, u=1, s=2$ and $t=1$. Namely, $g\left(u_{3}\right)=(1,0,1,1)$ and $g\left(u_{4}\right)=(-2,-1,-1,1)$. Next, we consider the case that $q \geqq 2$.

(I) Assume that $u=q$. By the inequalities (1), we have $s=2$ and $t=p+1$. Since

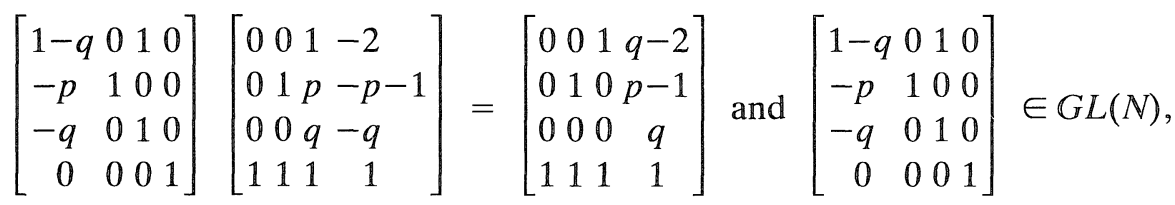

$q-2=1, p-1=1$ or $(q-2)+(p-1)=q$. Hence $q=3, p=2$ or $p=3$. Since

$\left[\begin{array}{rrrr}1 & 0 & -1 & 0 \\ 0 & 1 & -1 & 0 \\ 0 & 0 & -1 & 0 \\ 0 & 0 & 0 & 1\end{array}\right]\left[\begin{array}{rrrr}0 & 1 & 0 & -2 \\ 0 & 0 & 1 & -p-1 \\ 0 & 0 & 0 & -q \\ 1 & 1 & 1 & 1\end{array}\right]=\left[\begin{array}{rrrr}0 & 1 & 0 & q-2 \\ 0 & 0 & 1 & q-p-1 \\ 0 & 0 & 0 & q \\ 1 & 1 & 1 & 1\end{array}\right]$ and $\left[\begin{array}{rrrr}1 & 0 & -1 & 0 \\ 0 & 1 & -1 & 0 \\ 0 & 0 & -1 & 0 \\ 0 & 0 & 0 & 1\end{array}\right] \in G L(N)$, 
$q-2=1, q-p-1=1$ or $(q-2)+(q-p-1)=q$. Hence $q=3, q=p+2$ or $q=p+3$.

(i) When $q=3$, we have $p=1$ and $t=2$. Then the convex set $g\left(u_{3} u_{4}\right)$ contains the lattice point $(0,0,1,1)=\frac{2}{3} g\left(u_{3}\right)+\frac{1}{3} g\left(u_{4}\right)$. It contradicts the assumptions that $A L=\emptyset$.

(ii) When $p=2$ and $q=p+2$, we have g.c.d. $(p, q) \neq 1$.

(iii) when $p=2$ and $q=p+3$, we have $q=5$ and $t=3$. Hence $g\left(u_{3}\right)=(1,2,5,1)$ and $g\left(u_{4}\right)=(-2,-3,-5,1)$.

(iv) When $p=3$ and $q=p+2$, we have $p>\frac{q}{2}$.

(v) When $p=3$ and $q=p+3$, we have g.c.d. $(p, q) \neq 1$.

(II) Assume that $u<q$. By the inequalities (1), we have $s=1$ and $0<t \leqq p, u$. Since

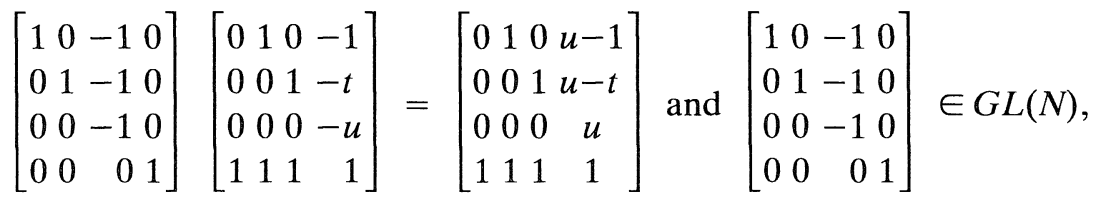

$u=1, u-1=1, u-t=1$ or $(u-1)+(u-t)=u$. Hence $u=t=1, u=2$ or $u=t+1$. Since

$$
\left[\begin{array}{cccc}
1-q & 0 & 1 & 0 \\
-p & 1 & 0 & 0 \\
-q & 0 & 1 & 0 \\
0 & 0 & 0 & 1
\end{array}\right]\left[\begin{array}{lllr}
0 & 0 & 1 & -1 \\
0 & 1 & P & -t \\
0 & 0 & q & -u \\
1 & 1 & 1 & 1
\end{array}\right]=\left[\begin{array}{lllc}
0 & 0 & 1 & q-u-1 \\
0 & 1 & 0 & p-t \\
0 & 0 & 0 & q-u \\
1 & 1 & 1 & 1
\end{array}\right],\left[\begin{array}{cccc}
1-q & 0 & 1 & 0 \\
-p & 1 & 0 & 0 \\
-q & 0 & 1 & 0 \\
0 & 0 & 0 & 1
\end{array}\right] \in G L(N)
$$

and $0 \leqq p-t=p \frac{u}{q}-t+\left(1-\frac{u}{q}\right) p<\left(1-\frac{u}{q}\right) p=(q-u) \frac{p}{q}<q-u$, we have $q-$ $u=1, q-u-1=1, p-t=1$ or $(q-u-1)+(p-t)=q-u$. Hence $q=u+1$, $q=u+2$ or $p=t+1$.

(i) When $u=t=1$ and $q=u+1$, we have $q=2$ and $p=1$. Hence $g\left(u_{3}\right)=$ $(1,1,2,1)$ and $g\left(u_{4}\right)=(-1,-1,-1,1)$.

(ii) When $u=t=1$ and $q=u+2$, we have $q=3$ and $p=1$. Then $g\left(u_{3} u_{4}\right)$ contains the lattice point $(0,0,1,1)=\frac{1}{2} g\left(u_{3}\right)+\frac{1}{2} g\left(u_{4}\right)$.

(iii) When $u=t=1$ and $p=t+1$, we have $p=2$ and $q \geqq 5$. Since

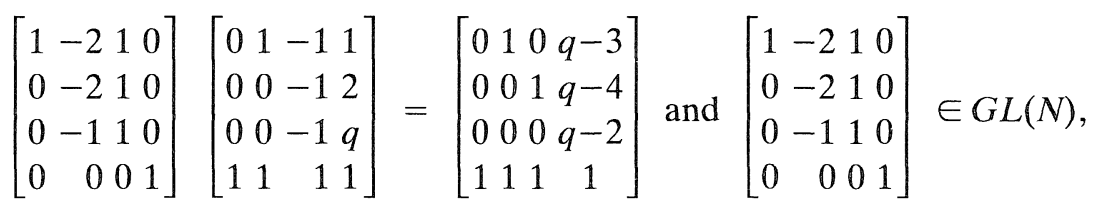
we have $q-2=1, q-3=1, q-4=1$ or $(q-3)+(q-4) \in(q-2) Z$. Hence $q=5$, because $q \geqq 5$. Therefore, $g\left(u_{3}\right)=(1,2,5,1)$ and $g\left(u_{4}\right)=(-1,-1,-1,1)$.

(iv) when $u=2$ and $q=u+1$, we have $q=3, p=1$ and $t=1$, by the third inequality in (1). Hence $g\left(u_{3}\right)=(1,1,3,1)$ and $g\left(u_{4}\right)=(-1,-1,-2,1)$.

(v) When $u=2$ and $q=u+2$, we have $q=4, p=1$ and $t=1$, by the third 
inequality in (1). Then $g\left(u_{3} u_{4}\right)$ contains the lattice point $(0,0,1,1)=\frac{1}{2} g\left(u_{3}\right)+\frac{1}{2} g\left(u_{4}\right)$. (vi) When $u=2$ and $p=t+1$, we have $t=1$ or 2 , because $0<t \leqq u$. Suppose that $t=2$. Then $g\left(u_{1} u_{4}\right)$ contains the lattice point $(0,-1,-1,1)=\frac{1}{2} g\left(u_{1}\right)+\frac{1}{2} g\left(u_{4}\right)$. Hence $t=1, g\left(u_{4}\right)=(-1,-1,-2,1), p=2$ and $q \geqq 5$. Since

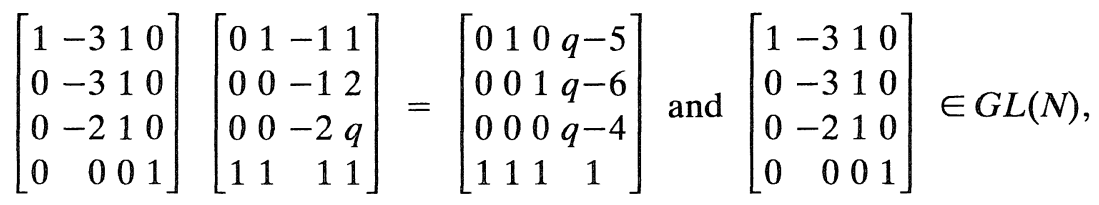

we have $q-4=1, q-5=1, q-6=1$ or $(q-5)+(q-6) \in(q-4) Z$. Hence $q=5$ or $q=7$, because $q \geqq 5$ and g.c.d. $(2, q)=1$. If $q=5$, then $g\left(u_{3}\right)=(1,2,5,1)$. If $q=7$, then $g\left(u_{3}\right)=(1,2,7,1)$.

(vii) When $u=t+1$ and $q=u+1$, we have $q \geqq 3$. By the third inequality in (1), we have $(q-2) q \leqq p(q-1)+q$. Hence $p \geqq q-2 q /(q-1)=q-(2+2 /$ $(q-1))$. Therefore, $q-p \leqq 3$ with the equality holds only if $q=3$. When $q=3$, we have $u=2, t=1$ and $p=1$, because $p \leqq \frac{q}{2}$. Hence $g\left(u_{3}\right)=(1,1,3,1)$ and $g\left(u_{4}\right)=(-1,-1,-2,1)$. When $q>3$, we have $q-p \leqq 2$. Then since $q-2 \leqq p \leqq \frac{q}{2}$, we have $q \leqq 4$. Hence $q=4$ and $p=2$. However, then g.c.d. $(p, q) \neq 1$.

(viii) when $u=t+1$ and $q=u+2$, we have $q \geqq 4$. Hence $p-t=1$, because $0 \leqq p-t<q-t=3$ and g.c.d. $(p-t, q-u)=1$. Then $\frac{q}{2} \geqq p=t+1=q-2$. Hence $q=4$ and $p=2$. However, then g.c.d. $(p, q) \neq 1$.

(ix) When $u=t+1=p$, we have $p \geqq 2$. If $p=2$, then $u=2$. This case was already considered in (vi). Therefore, we may assume that $p \geqq 3$. By the third inequality in (1), we have $(p-1) q \leqq p^{2}+q$. Hence $p^{2} \geqq(p-2) q \geqq 2(p-2) p$. Therefore, $p=3$ or $p=4$. When $p=3$, we have $u=3, t=2$ and $q=7$ or $q=8$, because g.c.d. $(p, q)=1, p \leqq \frac{q}{2}$ and $p^{2} \geqq(p-2) q$. If $q=8$, then $g\left(u_{0} u_{1} u_{3} u_{4}\right)$ contains the lattice point $(0,0,1,1)=\frac{1}{7} g\left(u_{0}\right)+\frac{1}{7} g\left(u_{1}\right)+\frac{2}{7} g\left(u_{3}\right)+\frac{3}{7} g\left(u_{4}\right)$. If $q=7$, then $g\left(u_{3}\right)=(1,3,7,1)$ and $g\left(u_{4}\right)=(-1,-2,-3,1)$. When $p=4$, we have $q=8$, because $p \leqq \frac{q}{2}$ and $p^{2} \geqq(p-2) q$. However, then g.c.d. $(p, q) \neq 1$.

q.e.d.

For the cases $(1-2)$ through (3) in $\$ 2$, it is easier to obtain similar propositions as Propositions 3.3 and 3.4, by Lemmas 3.1 and 3.2. Hence we only give a list of representaives of all pairs in $\widetilde{\mathscr{E}}_{0}^{3}$ below. We denote by $G(\sigma)$ the minimal set of generators of $\sigma$ which are primitive elements in $N=Z^{4}$.

$(1-1)$

1. $G(\sigma)=\{(1,0,0,0),(0,1,0,0),(0,0,1,0),(0,0,0,1)\}$. See [9, Table 2.2], for $u_{0}$.

2. $G(\sigma)=\{(0,0,0,1), \quad(1,0,0,1), \quad(0,1,0,1), \quad(1,1,2,1)\} \quad$ and $\quad u_{0}=\frac{1}{2}(1,1,1,2)$, $\frac{1}{3}(1,1,1,3), \frac{1}{12}(5,3,4,12), \frac{1}{8}(3,2,2,8), \frac{1}{20}(7,5,4,20), \frac{1}{16}(5,4,2,16), \frac{1}{9}(3,2,1,9), \frac{1}{5}(2,1,1,5)$, $\frac{1}{13}(4,3,1,13), \frac{1}{11}(5,2,3,11), \frac{1}{14}(5,3,2,14), \frac{1}{16}(7,3,4,16), \frac{1}{19}(4,7,3,19)$ or $\frac{1}{24}(5,9,4,24)$.

3. $G(\sigma)=\{(0,0,0,1), \quad(1,0,0,1), \quad(0,1,0,1), \quad(1,1,3,1)\} \quad$ and $u_{0}=\frac{1}{4}(2,1,2,4)$, $\frac{1}{7}(3,2,1,7), \frac{1}{5}(3,1,2,5), \frac{1}{9}(5,2,3,9), \frac{1}{6}(2,3,3,6), \frac{1}{9}(3,4,3,9)$ or $\frac{1}{2}(1,1,2,2)$. 
4. $G(\sigma)=\{(0,0,0,1),(1,0,0,1),(0,1,0,1),(1,1,4,1)\}$ and $u_{0}=\frac{1}{2}(1,1,2,2)$.

5. $G(\sigma)=\{(0,0,0,1), \quad(1,0,0,1), \quad(0,1,0,1), \quad(1,2,5,1)\} \quad$ and $\quad u_{0}=\frac{1}{4}(2,3,5,4)$, $\frac{1}{5}(2,3,5,5), \frac{1}{2}(1,1,1,2)$ or $\frac{1}{3}(1,1,1,3)$.

6. $G(\sigma)=\{(0,0,0,1),(1,0,0,1),(0,1,0,1),(1,2,7,1)\}$ and $u_{0}=\frac{1}{2}(1,1,2,2)$.

7. $G(\sigma)=\{(0,0,0,1),(1,0,0,1),(0,1,0,1),(1,3,7,1)\}$ and $u_{0}=\frac{1}{2}(1,2,3,2)$.

8. $G(\sigma)=\{(0,0,0,1),(1,0,0,1),(0,1,0,1),(1,3,8,1)\}$ and $u_{0}=\frac{1}{2}(1,2,4,2)$.

9. $G(\sigma)=\{(0,0,0,1),(1,0,0,1),(0,1,0,1),(1,3,10,1)\}$ and $u_{0}=\frac{1}{2}(1,2,5,2)$.

10. $G(\sigma)=\{(1,0,0,1),(0,1,0,1),(0,0,1,1),(-1,-1,-1,1)\}$ and $u_{0}=(0,0,0,1)$.

11. $G(\sigma)=\{(1,0,0,1),(0,1,0,1),(1,1,2,1),(-1,-1,-1,1)\}$ and $u_{0}=(0,0,0,1)$.

12. $G(\sigma)=\{(1,0,0,1),(0,1,0,1),(1,1,3,1),(-1,-1,-2,1)\}$ and $u_{0}=(0,0,0,1)$.

13. $G(\sigma)=\{(1,0,0,1),(0,1,0,1),(1,2,5,1),(-1,-1,-1,1)\}$ and $u_{0}=(0,0,0,1)$.

14. $G(\sigma)=\{(1,0,0,1),(0,1,0,1),(1,2,5,1),(-1,-1,-2,1)\}$ and $u_{0}=(0,0,0,1)$.

15. $G(\sigma)=\{(1,0,0,1),(0,1,0,1),(1,2,5,1),(-2,-3,-5,1)\}$ and $u_{0}=(0,0,0,1)$.

16. $G(\sigma)=\{(1,0,0,1),(0,1,0,1),(1,2,7,1),(-1,-1,-2,1)\}$ and $u_{0}=(0,0,0,1)$.

17. $G(\sigma)=\{(1,0,0,1),(0,1,0,1),(1,3,7,1),(-1,-2,-3,1)\}$ and $u_{0}=(0,0,0,1)$. $(1-2)$

1. $G(\sigma)=\{(1,0,0,1),(0,1,0,1),(-1,-1,0,1),(1,0,1,1)\}$ and $u_{0}=\frac{1}{2}(0,0,1,2)$, $\frac{1}{3}(-1,-1,1,3), \frac{1}{4}(-1,-1,2,4)$ or $\frac{1}{6}(-1,-1,2,6)$.

2. $G(\sigma)=\{(2,0,0,1),(0,1,0,1),(-1,-1,0,1),(1,0,1,1)\}$ and $u_{0}=\frac{1}{2}(1,0,1,2)$.

3. $G(\sigma)=\{(1,0,0,1),(0,1,0,1),(-1,-1,0,1),(1,2,3,1)\}$ and $u_{0}=\frac{1}{3}(2,2,3,3)$.

4. $G(\sigma)=\{(1,0,0,1),(0,1,0,1),(-1,-1,0,1),(0,0,2,1)\}$ and $u_{0}=(0,0,1,1)$.

5. $G(\sigma)=\{(2,0,0,1),(0,1,0,1),(-1,-1,0,1),(-1,0,2,1)\}$ and $u_{0}=(0,0,1,1)$.

6. $G(\sigma)=\{(1,0,0,1),(0,1,0,1),(-1,-1,0,1),(-1,0,3,1)\}$ and $u_{0}=(0,0,1,1)$. $(2-1)$

1. $G(\sigma)=\{(0,0,0,1),(1,0,0,1),(0,1,0,1),(1,0,1,1),(-1,0,-1,1)\}$ and $u_{0}=$ $\frac{1}{3}(1,1,0,3), \frac{1}{4}(1,1,0,4)$ or $\frac{1}{6}(1,2,0,6)$.

2. $G(\sigma)=\{(0,0,0,1),(1,0,0,1),(0,1,0,1),(1,0,1,1),(0,0,-1,1)\}$ and $u_{0}=$ $\frac{1}{3}(1,1,0,3)$ or $\frac{1}{4}(1,2,0,4)$.

3. $G(\sigma)=\{(0,0,0,1),(1,0,0,1),(0,1,0,1),(1,1,2,1),(0,0,-1,1)\}$ and $u_{0}=$ $\frac{1}{4}(1,1,0,4)$.

4. $G(\sigma)=\{(0,0,0,1),(1,0,0,1),(0,1,0,1),(1,1,2,1),(-1,-1,-2,1)\}$ and $u_{0}=$ $\frac{1}{4}(1,1,0,4)$.

5. $G(\sigma)=\{(0,0,0,1),(1,0,0,1),(0,1,0,1),(1,1,3,1),(1,-1,-3,1)\}$ and $u_{0}=$ $\frac{1}{3}(1,1,0,3)$.

6. $G(\sigma)=\{(1,0,0,1),(0,1,0,1),(-1,-1,0,1),(0,0,1,1),(0,0,-1,1)\}$ and $u_{0}=$ $(0,0,0,1)$.

7. $G(\sigma)=\{(1,0,0,1),(0,1,0,1),(-1,-1,0,1),(0,0,1,1),(1,1,-1,1)\}$ and $u_{0}=$ $(0,0,0,1)$.

8. $G(\sigma)=\{(1,0,0,1),(0,1,0,1),(-1,-1,0,1),(0,0,1,1),(2,0,-1,1)\}$ and $u_{0}=$ $(0,0,0,1)$.

9. $G(\sigma)=\{(1,0,0,1),(0,1,0,1),(-1,-1,0,1),(1,2,3,1),(-1,-2,-3,1)\}$ and $u_{0}=(0,0,0,1)$. 
$(2-2-1)$

1. $G(\sigma)=\{(1,0,0,1),(-1,0,0,1),(0,1,1,1),(0,1,-1,1)\}$ and $u_{0}=\frac{1}{2}(0,1,0,2)$.

2. $G(\sigma)=\{(1,0,0,1),(-1,0,0,1),(1,1,2,1),(-1,1,-2,1)\}$ and $u_{0}=\frac{1}{2}(0,1,0,2)$.

3. $G(\sigma)=\{(1,0,0,1),(-1,0,0,1),(0,2,1,1),(0,2,-1,1)\}$ and $u_{0}=(0,1,0,1)$.

4. $G(\sigma)=\{(1,0,0,1),(-1,0,0,1),(1,2,2,1),(-1,2,-2,1)\}$ and $u_{0}=(0,1,0,1)$. $(2-2-2)$

1. $G(\sigma)=\{(1,0,0,1),(-1,0,0,1),(0,1,0,1),(0,-1,0,1),(0,0,1,1)\}$ and $u_{0}=$ $\frac{1}{2}(0,0,1,2)$.

2. $G(\sigma)=\{(1,0,0,1),(-1,0,0,1),(0,1,0,1),(0,-1,0,1),(1,1,2,1)\}$ and $u_{0}=$ $\frac{1}{2}(1,1,2,2)$.

3. $G(\sigma)=\{(1,0,0,1),(-1,0,0,1),(0,1,0,1),(0,-1,0,1),(0,0,2,1)\}$ and $u_{0}=$ $(0,0,1,1)$.

$(2-2-3)$

1. $G(\sigma)=\{(1,1,0,1),(0,0,1,1),(0,0,-1,1),(0,1,0,1),(1,0,0,1)\}$ and $u_{0}=$ $\frac{1}{2}(1,1,0,2)$.

2. $G(\sigma)=\{(-1,0,0,1),(1,0,1,1),(1,0,-1,1),(0,1,0,1),(0,-1,0,1)\}$ and $u_{0}=$ $(0,0,0,1)$.

3. $G(\sigma)=\{(-1,0,0,1),(1,1,2,1),(1,-1,-2,1),(0,1,0,1), \quad(0,-1,0,1)\} \quad$ and $u_{0}=(0,0,0,1)$.

4. $G(\sigma)=\{(-1,0,0,1),(1,0,1,1),(1,0,-1,1),(0,1,0,1),(1,-1,0,1)\}$ and $u_{0}=$ $(0,0,0,1)$.

$(2-2-4)$

1. $G(\sigma)=\{(0,-1,2,1),(0,2,0,1),(1,0,0,1),(-1,0,0,1)\}$ and $u_{0}=(0,0,1,1)$. $(2-3)$

1. $G(\sigma)=\{(1,0,0,1),(-1,0,0,1), \quad(0,2,0,1), \quad(0,0,1,1)\} \quad$ and $u_{0}=\frac{1}{2}(0,1,1,2)$, $\frac{1}{3}(0,3,1,3)$ or $\frac{1}{4}(0,4,1,4)$.

2. $G(\sigma)=\{(1,0,0,1),(-1,0,0,1),(0,3,0,1),(0,0,1,1)\}$ and $u_{0}=\frac{1}{2}(0,2,1,2)$.

3. $G(\sigma)=\{(1,0,0,1),(-1,0,0,1),(0,2,0,1),(1,1,2,1)\}$ and $u_{0}=\frac{1}{2}(1,1,2,2)$. $(2-4)$

1. $G(\sigma)=\{(0,0,2,1),(0,3,0,1),(1,0,0,1),(-1,0,0,1)\}$ and $u_{0}=(0,1,1,1)$.

(3)

1. $G(\sigma)=\{(0,0,0,1),(1,1,0,1),(1,0,0,1),(0,1,0,1),(0,0,1,1),(1,1,-1,1)\}$ and $u_{0}=\frac{1}{2}(1,1,0,2)$.

2. $G(\sigma)=\{(1,0,0,1),(-1,0,0,1),(0,1,0,1),(0,-1,0,1),(0,0,1,1),(0,0,-1,1)\}$ and $u_{0}=(0,0,0,1)$.

3. $G(\sigma)=\{(1,0,0,1),(-1,0,0,1),(0,1,0.1),(0,-1,0,1),(1,1,2,1),(-1,-1,-2,1)\}$ and $u_{0}=(0,0,0,1)$.

\section{References}

[1] Ishida M.-N., Torus embeddings and dualizing complexes, Tôhoku Math. J., 32 (1980), 111146.

[2] Ishii S., On isolated Gorenstein singularities, Math. Ann., 270 (1985), 541-554. 
[3] Ishii S. and Watanabe K., On Simple K3 singularities, Note appeared in the proceedings of the conference of Algebraic Geometry at Tokyo Metropolitan Univ., (1988), 20-31.

[4] Morrison D.R. and Stevens G., Terminal quotient singularities in dimension three and four, Proc. Amer. Math. Soc., 90 (1984), 15-20.

[5] Oka M., On the resolution of the hypersurface singularities, Complex analytic singularities (T. Suwa and P. Wagreigh, eds.), Advanced Studies in Pure Math., 8, Kinokuniya, Tokyo and North-Holland, Amsterdam, New York, Oxford, 1986, 405-436.

[6] Tsuchihashi H., Hypersurface sections of toric singularities, Kodai Math. J., 14 (1991), 210221.

[7] Watanabe K., Ishikawa T., Tachibana S. and Otsuka K., On tensor products of Gorenstein rings, J. Math. Kyoto U., 15 (1975) 387-395.

[8] Watanabe K., On plurigenera of normal isolated singularities II, Complex analytic singularities (T. Suwa and P. Wagreigh, eds.), Advanced Studies in Pure Math., 8, Kinokuniya, Tokyo and North-Holland, Amsterdam, New York, Oxford, 1986, 671-685.

[9] Yonemura T., On hypersurface simple K3 singularities, Tôhoku Math. J., 42 (1990) 351-380. 
